Pacific Journal of Mathematics

A NOTE ON ORDERINGS ON ALGEBRAIC VARIETIES 


\title{
A NOTE ON ORDERINGS ON ALGEBRAIC VARIETIES
}

\author{
M. E. Alonso
}

It was proven in [A-G-R] that if $V \subset \mathbf{R}^{n}$ is a surface and $\alpha$ a total ordering in its coordinate polynomial ring, $\alpha$ can be described by a half branch (i.e., there exists $\gamma(0, \varepsilon) \rightarrow V$, analytic, such that for every $f \in \mathbf{R}[V] \operatorname{sgn}_{\alpha} f=\operatorname{sgn} f(\gamma(t))$ for $t$ small enough). Here we prove (in any dimension) that the orderings with maximum rank valuation can be described in this way. Furthermore, if the ordering is centered at a regular point we show that the curve can be extended $C^{\infty}$ to $t=0$.

1. (1.0) Let $V$ be an algebraic variety over $\mathbf{R}$ and $\alpha$ an ordering in $K=\mathbf{R}(V)$. If $\alpha$ is described by a half-branch $\gamma:(0, \varepsilon) \rightarrow V$, no non-zero polynomial vanishes over $\gamma(t)$ for $t$ small enough. Consequently, if $V^{\prime}$ is birrationally equivalent to $V$ (i.e., $\mathbf{R}\left(V^{\prime}\right)=\mathbf{R}(V)$ ), $\alpha \cap \mathbf{R}\left[V^{\prime}\right]$ is also described by a curve in $V^{\prime}$.

(1.1) Proposition. Let $V$ be an algebraic variety over $\mathbf{R}$ and $n=\operatorname{dim} V$. If $\mathbf{R}[V]$ is an integral extension of $\mathbf{R}\left[x_{1}, \ldots, x_{n}\right]=\mathbf{R}[\underline{x}]$ and $\alpha$ an ordering on $\mathbf{R}[V]$ such that $\beta=\alpha \cap \mathbf{R}[\underline{x}]$ can be described by a half-branch, then the same holds true for $\alpha$.

Proof. By our previous remark (1.0) we can suppose $V$ is a hypersurface. Thus $\mathbf{R}[V]=\mathbf{R}\left[\underline{x}, x_{n+1}\right](P)$ where $P \in \mathbf{R}[\underline{x}]\left[x_{n+1}\right]$ is a monic polynomial in $x_{n+1}$. Let $\delta$ be the discriminant of $P$ and $\pi: V \rightarrow \mathbf{R}^{n}$ the projection on the first $n$-coordinates. Then the restriction

$$
\pi_{\mid}: V \backslash \pi^{-1}(\delta=0) \rightarrow \mathbf{R}^{n} \backslash\{\delta=0\}
$$

has finite fibers with constant cardinal over every connected component. Moreover, by the implicit function theorem, $\pi_{1}$ is an analytic diffeomorphism from every connected component of $V \backslash \pi^{-1}(\delta=0)$ onto someone of $\mathbf{R}^{n} \backslash\{\delta=0\}$.

Let $\gamma:(0, \varepsilon) \rightarrow \mathbf{R}^{n}$ be the curve describing $\beta$. The connected components $C_{1}, \ldots, C_{p}$ of $\mathbf{R}^{n} \backslash\{\boldsymbol{\delta}=0\}$ are open semi-algebraic sets, and we can write

$$
C_{i}=\bigcup_{j=1}^{q}\left\{f_{i j 1}>0, \ldots, f_{i j r}>0\right\}, \quad f_{i j l} \in \mathbf{R}[\underline{x}]
$$


As $\gamma$ describes the ordering in $\mathbf{R}[\underline{x}]$ and the $C_{i}$ 's are pairwise disjoint, for $t$ small enough, $f_{i j l}(\gamma(t))$ does not change the sign and $\gamma(t)$ is contained in a unique $C_{i_{0}}$. We put $C=C_{i_{0}}$.

Let $D_{1}, \ldots, D_{s}$ (we shall see below that $s$ is not zero) be the connected components of $V \backslash \pi^{-1}\{\delta=0\}$ diffeomorphic to $C$ via $\pi$. We claim that

$$
s=\text { number of extensions of } \beta \text { to } R(V) \text {. }
$$

By construction $s$ is the number of roots of $P\left(\underline{x}, x_{n+1}\right)$ for every $\underline{x} \in C$. On the other hand, the number of extensions of $\beta$ to $\mathbf{R}(V)$ coincides with the number of roots of $P \in \mathbf{R}(\underline{x})\left[x_{n+1}\right]$ in a real closure of $(\mathbf{R}(\underline{x}), \beta)$ (see [Pr] 3.12). We shall prove now the latter is also the number of real roots of $P\left(\underline{x}, x_{n+1}\right)$ for $\underline{x} \in C$.

Let $S=\left\{P_{0}, \ldots, P_{l}\right\} \mathbf{R}(\underline{x})\left[x_{n+1}\right]$ be the standard Sturm sequence of

$$
P\left(\underline{x}, x_{n+1}\right)=x_{n+1}^{m}+a_{1} x_{n+1}^{m-1}+\cdots+a_{m}, \quad M=1+m+\sum_{i=1}^{m} a_{i}^{2}
$$

and $\Delta$ the product of all numerators and denominators of the non-zero coefficients of the polynomials in $x_{n+1}$ used in the construction of $S$. In this situation, by Artin's specialization theorem there exists $\underline{x}_{0} \in \mathbf{R}^{n}$ such that

(a) $f_{i_{0} h}\left(\underline{x}_{0}\right)>0, \Delta\left(\underline{x}_{0}\right) \neq 0$, some $j=1, \ldots, q$, all $h=1, \ldots, r$

(b) $\operatorname{sgn}_{\beta} P_{k}( \pm M)=\operatorname{sgn}_{\mathbf{R}} P_{k}\left(\underline{x}_{0}, \pm M\left(\underline{x}_{0}\right)\right), k=0, \ldots, l$.

By (a), $\underline{x}_{0} \in C$ and $S_{x_{0}}=\left\{P_{1}\left(\underline{x}_{0}\right), \ldots, P_{l}\left(\underline{x}_{0}\right)\right\}$ is the standard Sturm sequence of $P\left(\underline{x}_{0}, x_{n+1}\right)$. By (b) the number of sign changes of $S_{\underline{x}_{0}}$ and $S$ coincides. Then the claim is proven.

Now, let us denote by $\gamma_{k}=\left(\pi_{\mid D_{k}}\right)^{-1} \circ \gamma, k=1, \ldots, l$ the liftings of $\gamma$. Then it is easy to prove:

$\left(\mathrm{a}^{\prime}\right)$ If $f \in \mathbf{R}[V] \backslash\{0\}, f\left(\gamma_{k}(t)\right) \neq 0$ and its sign does not change for $t$ small enough. Consequently every $\gamma_{k}$ defines an ordering that we call $\alpha_{k}$.

(b') If $k \neq k^{\prime}, \alpha_{k} \neq \alpha_{k^{\prime}}$.

From the remarks above, $\alpha$ must be equal to some $\alpha_{k}$, hence it is described by the corresponding $\alpha_{k}$.

2. (2.0) Let $K$ and $\Delta$ be ordered fields and $p: K \rightarrow \Delta, \infty$ a place such that for $x$ positive, $p(x)$ is not negative. Then we define a signed place $\hat{p}: K \rightarrow \Delta \cup\{+\infty, \infty\}=\Delta, \pm \infty$ in the following way:

$$
\hat{p}(x)=p(x) \text { if } p(x) \neq \infty ; \quad \hat{p}(x)=\operatorname{sign}(x) \cdot \infty \quad \text { if } p(x)=\infty .
$$

Now assume $K$ is the function field of a real algebraic variety $V$, and $\alpha$ an ordering in $K$. A point $O \in V$ is the center of $\alpha$ in $V$ if the real valued canonical place $p_{\alpha}$ associated to $\alpha$ (see [B] Chap. VII) is finite over 
$\mathbf{R}[V]$ and the ideal of $O$ is the center of $p_{\alpha}$ in $\mathbf{R}[V]$. In that case, every function positive at $O$ is positive in $\alpha$, and if $\alpha$ is described by $\gamma$, then $\lim _{t \rightarrow 0} \gamma(t)=O$.

We are interested in the case when the rank of $p_{\alpha}$ is maximum (i.e., it coincides with the dimension of $V$ ). In this situation the decomposition of $p_{\alpha}$ in rank 1 places is

$$
K=K \stackrel{\theta_{n-1}}{\rightarrow} K_{n-1}, \infty \rightarrow \cdots \rightarrow \mathbf{R}, \infty
$$

where $K_{j}$ is a function field over $\mathbf{R}$ of dimension $j$. Then it is possible to define uniquely orderings in $K_{j}(j=1, \ldots, r)$ such that, considering $\alpha$ in $K$, all places verify the compatibility conditions. Thus we consider the associated signed places $\hat{\theta}_{j}: K_{j} \rightarrow K_{j-1}, \pm \infty$ (see [B] Chap. VIII), to get a decomposition of $\hat{p}_{\alpha}$ in rank 1 signed places.

(2.1) Proposition. If $p_{\alpha}$ has a maximum rank, $\alpha$ can be described by a half-branch.

Proof. The proof goes by induction. If $n=1$, by 1.1 and 1.0 we can suppose $K=\mathbf{R}(x), \alpha$ centered at $x=0$, and $x>{ }_{\alpha} 0$. Then, there is a unique ordering with this property (i.e., making $x$ infinitesimal with respect to $\mathbf{R}$ and positive), and it is described by the curve $\gamma(t)=t$.

In the general situation we can choose $\zeta_{1}, \ldots, \zeta_{n-1}, \zeta_{n}$ in $K$ such that $\theta_{n-1}\left(\zeta_{1}\right), \ldots, \theta_{n-1}\left(\zeta_{n}\right) \in K_{n-1}$ and:

(i) $\theta_{n-1}\left(\zeta_{1}\right), \ldots, \theta_{n-1}\left(\zeta_{n-1}\right)$ are algebraically independent.

(ii) $\zeta_{1}, \ldots, \zeta_{n}$ are algebraically independent

(iii) $p_{\alpha}\left(\zeta_{l}\right)=0(i=1, \ldots, n)$.

Since $K$ is the quotient field of the integral closure of $B=$ $\mathbf{R}\left[\zeta_{1}, \ldots, \zeta_{n-1}, \zeta_{n}\right]$ we can suppose $K=q \cdot f(B)$ by 1.1 . Then the kernel of $\theta_{n-1 \mid}: B \rightarrow K_{n-1}$ is an height one prime ideal and hence it is generated by some $F \in B$. The field $K_{n-1}$ is the function field of the hypersurface $\{F=0\}$. Moreover we may assume $F>{ }_{\alpha} 0$.

Let us consider, according to 2.0 , the ordering $\beta$ associated to $r=\theta_{0} \circ \cdots \circ \theta_{n-2}$ in $K_{n-1}$. Then $p_{\beta}=r$ and $\beta$ is centered at $\underline{0}=$ $(0, \ldots, 0)$ which belongs to the hypersurface. Consequently, for every $f \in B$ we have:

(2.1.1) if $f(\underline{0})=p_{\alpha}(f) \neq 0$, then $\operatorname{sgn}_{\alpha} f=\operatorname{sgn} f(\underline{0})$

if $\theta_{n-1}(f) \neq 0, \operatorname{sgn}_{\alpha} f=\operatorname{sgn}_{\beta} \bar{f}$, where $\bar{f}$ is $f+(F)$ if $\theta_{n-1}(f)=0$ and $f=u \cdot F^{r}$ with g.c.d $(u, F)=1$, then $\operatorname{sgn}_{\alpha} f=\operatorname{sgn}_{\alpha} u=\operatorname{sgn}_{\beta} \bar{u}$. 
Now we need a lemma:

(2.2) LEMMA. Let $H=\{F(\underline{x})=0\}$ be a real irreducible hypersurface in $\mathbf{R}^{n}$ and $\beta$ a rank $(n-1)$ ordering in $H$ (i.e., in $\mathbf{R}[\underline{x}] /(F)$ ) centered at the point $\underline{0}$ and described by $\gamma:(0, \varepsilon) \rightarrow H$. Then, there is not more than one ordering $\alpha$ in $\mathbf{R}[\underline{x}]$ making $F$ infinitesimal and positive, and inducing $\beta$ in $\mathbf{R}[\underline{x}] /(F)$. Moreover $\alpha$ can be described by a half-branch.

Proof. The first claim is an easy consequence of 2.1.1.

Next, as $p_{\beta}$ has rank $n-1, p_{\beta}$ is discrete and its value group is isomorphic to $Z \oplus \stackrel{n-1}{\cdots} \oplus Z$, lexicographically ordered. Let $\bar{h} \in$ $\mathbf{R}[\underline{x}] /(F)$ have value $\left(a_{1}, \ldots, a_{n-1}\right)$ with $a_{1} \geq 1$ (notice that this is possible because the valuation ring of $p_{\beta}$ contains $\mathbf{R}[\underline{x}] /(F)$ ), and put $\psi(t)=h(\gamma(t))$. Since $p_{\beta}(\bar{h})=0, h(\underline{0})=0$ and $\lim _{t \rightarrow 0} \psi(t)=0, \psi$ is analytic in $(0, \varepsilon)$. Now we define the analytic curve:

$$
\gamma^{*}:(0, \varepsilon) \rightarrow \mathbf{R}^{n}: t \mapsto\left(\gamma_{i}(t)+c_{i} e^{-1 / \psi(t)^{2}}\right) \quad i=1, \ldots, n
$$

where the $c_{i}$ 's will be determined later.

Thus, the result follows from the statements (a) and (b) below.

(a) For any $c_{i}$ 's, if $G \in \mathbf{R}[x]$ is positive along $\gamma$, so is along $\gamma^{*}$.

(b) There is $\left(c_{1}, \ldots, c_{n}\right) \in \mathbf{R}^{n}$ such that $F\left(\gamma^{*}(t)\right)>0$ for $t$ small enough.

To prove (a) we first write:

$$
G\left(\gamma^{*}(t)\right)=G(\gamma(t))+m(t) e^{-1 / \psi(t)^{2}}
$$

where $m(t)$ is a polynomial in $\gamma_{1}(t), \ldots, \gamma_{n}(t)$ and $e^{-1 / \psi(t)^{2}}$. On the other hand, looking at the value of $\bar{h}$, for large $m \in N$ we know that $\bar{h}^{m} / \bar{G}$ $(\bar{G}=G+(F) \in \mathbf{R}[\underline{x}] /(F))$ is infinitesimal in $\beta$ w.r.t. $\mathbf{R}$ and so, $1-$ $\bar{h}^{m} / \bar{G}>{ }_{\beta} 0$. Since $\bar{G}$ is positive in $\beta$, taking an even $m$ we have $\bar{G}>{ }_{\beta} \bar{h}^{m}$ $>_{\beta} 0$. Hence $G(\gamma(t))>_{\beta} \psi(t)^{m}>_{\beta} 0$ for small $t$ enough, what implies $\lim _{t \rightarrow 0} e^{-1 / \psi(t)^{2}} / G(\gamma(t))=0$. Thus, we get (a) after dividing in 2.2.1 by $G(\gamma(t))$ and taking the limit when $t \rightarrow 0$.

For (b), we take the Taylor expansion of $F$ at $\gamma(t)$ and compute it at $\gamma^{*}(t)$ :

$$
\begin{aligned}
F\left(\gamma^{*}(t)\right)= & \sum_{i=1}^{n} \frac{\partial F(\gamma(t))}{\partial x_{i}} c_{i} e^{-1 / \psi(t)^{2}} \\
& +\sum_{i, j} \frac{\partial^{2} F(\gamma(t))}{\partial x_{i} \partial x_{j}} c_{i} c_{j} e^{-2 / \psi(t)^{2}}+\cdots
\end{aligned}
$$


As $\partial F / \partial x_{i} \notin(F)$ for some $i$, we have $c_{i}=\operatorname{sgn}_{\beta}\left(\partial F / \partial x_{i}\right)(= \pm 1 \neq 0)$ and we take $c_{j}=0$ for $j \neq i$. Then, $\beta$ being described by $\gamma$ :

$$
H(t)=\sum_{i=1}^{n} \frac{\partial F(\gamma(t))}{\partial x_{i}} c_{i}>0, \text { for small } t .
$$

Again we have $\lim _{t \rightarrow 0} e^{-1 / \psi(t)^{2}} / H(t)=0$. Then, dividing in 2.2 .2 by $H(t)$, we find $F\left(\gamma^{*}(t)\right) / H(t)>0$, hence $F\left(\gamma^{*}(t)\right)>0$, for small $t$.

(2.3) REMARK. Looking at the class of the curve $\gamma$ at 0 , we see that if $O \in \operatorname{Reg} H$, and $\gamma$ can be extended $C^{\infty}$ to $t=0$, the same holds true for $\gamma^{*}$.

(2.4) REMARK. Notice that 2.2 and 2.3 hold also true if we replace $\mathbf{R}^{n}$ by an algebraic variety $V$ with $O \in \operatorname{Reg} V$. In fact the same proof applies, by taking a regular system of parameters at $O$ in the place of $x_{1}, \ldots, x_{n}$.

(2.5) Application. As an example of the constructibility of the proof of 2.1 we determine the curves describing the rank 2 orderings in $\mathbf{R}^{2}$ (see [A-G-R]).

Firstly, after changes $x \rightarrow \pm(x \pm a)^{ \pm 1}, y \rightarrow \pm(y \pm b)^{ \pm 1}$, we can suppose $(0,0)$ is the center of the ordering $\alpha$ and $x>_{\alpha} 0, y>_{\alpha} 0$. Assume the divisor $w$ which specializes $p_{\alpha}$ is centered in $\mathbf{R}[x, y]$ at $F(x, y)=0$, and $x=t^{n}, y=a_{1} t^{n_{1}}+\cdots\left(n \leq n_{1}\right), t>0$, is a primitive parametrization of the half-branch describing the corresponding ordering in $\mathbf{R}[x, y] /(F)$. According to the above parametrization and looking at the proof of 2.2, we may choose $h(x)=x, c_{1}=0$ and $c_{2}= \pm 1$ in the proof of 2.2, and we get a half-branch describing $\alpha$ of the form:

$$
\gamma(t)=\left(t^{n}, \pm e^{-1 / t^{2 n}}+a_{1} t^{n_{1}}+\cdots\right)
$$

Now assume that the prime divisor $w$ is centered at the maximal ideal, $(x, y)$. Let us call $v$ the valuation corresponding to $p_{\alpha}$. Following Abhyankar [A], after a finite number of quadratic transforms along $w$ we get the previous situation. In fact, we call $A_{0}=\mathbf{R}[x, y]$ and, if $v(x) \leq v(y)$ (so $w(x) \leq w(y)$ ) we put: $r_{0}=p_{\alpha}(y / x), y_{1}=\left(y-r_{0} x\right) / x, x_{1}=x$ and $A_{1}=A_{1}\left[x_{1}, y_{1}\right]$. Repeating this procedure we end at $A_{s}=A_{s-1}\left[x_{s}, y_{s}\right]=$ $\mathbf{R}\left[x_{s}, y_{s}\right]$ such that, the center of $w$ in $A_{s}$ is 1-dimensional, and $w$ is centered at $\left(x_{s-1}, y_{s-1}\right)$ in $A_{s-1}$. We have, say,

$$
y_{s}=\left(y_{s-1}-r_{s-1} x_{s-1}\right) / x_{s-1}
$$


and $x_{s}=x_{s-1}$. Hence $w\left(x_{s}\right)=w\left(x_{s-1}\right)>0$ and $M_{w} \cap A_{s}=\left(x_{s}\right)$. Thus, according to the proof of 2.2, the half-branch $x_{s}= \pm e^{-1 / t^{2}}, \quad y_{s}=t$ describes the ordering in $A_{s}$. Hence, going backwards in the quadratic transformations, it follows easily that the ordering $\alpha$ can be described by a curve

$$
\left(P\left(t, e^{-1 / t^{2}}\right), Q\left(t, e^{-1 / t^{2}}\right)\right)
$$

for some polynomials $P$ and $Q$.

3. (3.0) We finish this note with some considerations about the class at $t=0$ of the $\gamma$ 's describing orderings (see also [R] §3). To start with notice that any algebraically independent power series $x_{1}(t), \ldots, x_{n}(t)$, describe an ordering in $\mathbf{R}[x]$. Then by [An] the set of such orderings is dense in the space of all orderings endowed with the Harrison Topology $[\mathbf{H}]$. Moreover, the valuations associated to these orderings are discrete of rank one. Hence the orderings with maximum rank valuation, cannot be described by curves which are analytic at $t=0$ unless the variety is a curve. So, the best result we can expect is the following:

(3.1) Proposition. If $V \subset \mathbf{R}^{n}$ is an algebraic variety an $\alpha$ an ordering centered at $0=(0, \ldots, 0) \operatorname{Reg} V$, with associated valuation of maximum rank, there is a half-branch describing $\alpha$ which can be extended $C^{\infty}$ (but not analytically) to $t=0$. Furthermore the set of orderings of $\mathbf{R}[V]$ described by half-branches $C^{\infty}$ at $t=0$ but not by analytic ones, is dense in the space of orderings.

Proof. The proof goes by induction on $d=\operatorname{dim} V$. If $d=1$, the valuation associated to the ordering $\alpha$ is discrete, has rank one, and the ordering is described by the unique branch of $V$ through 0 :

$$
\left(t, u_{2}(t), \ldots, u_{n}(t)\right)
$$

where each $u_{i}(t)$ is analytic and the choice $t>0$ or $t<0$.

In the general case, set $\hat{p}_{\alpha}=p$ and consider again

$$
K=\mathbf{R}(V) \stackrel{q}{\rightarrow} K_{n-1}, \pm \infty \stackrel{r}{\rightarrow} \mathbf{R}, \pm \infty, \quad p=r \circ q
$$

the decomposition of $p$ in signed places of rank one.

As we did in 2.1 we can find an (affine) algebraic variety $V_{1}$ and $\pi$ : $V_{1} \rightarrow V$ birational morphism such that the center of $q$ in $V_{1}$, say $H_{1}$, has dimension $d-1$. By means of Hironaka's desingularization I [Hi] we may assume $V_{1}$ is smooth. Then by Hironaka's desingularization II (loc. cit), we find $\tilde{V}$ and $\tilde{\pi}: \tilde{V} \rightarrow V_{1}$, a proper birrational map such that $\tilde{\pi}^{-1}\left(H_{1}\right)$ is 
a normal crossing divisor. Let $\tilde{0}$ be the center of $p$ in $\tilde{V}$ and $\tilde{H}$ the center of $q$. Since the valuation ring of $q, \mathbf{R}\left[V_{1}\right]_{\mathscr{J}\left(H_{1}\right)}$, dominates $\mathbf{R}[\tilde{V}]$ and $\tilde{H}$ lies over $H_{1}$, we have $K_{n-1}=q f \cdot \tilde{H}$ and the center of $r$ in $\tilde{H}$ is $\tilde{0}$.

We call $\beta$ the ordering in $K_{n-1}$ corresponding to the precedent decomposition (i.e. $\hat{p}_{\beta}=r$ ). Since $r$ has maximum rank, by our inductive hypothesis the ordering $\beta \cap \mathbf{R}[\tilde{H}]$ can be described by $\gamma:(0, \varepsilon) \rightarrow \tilde{H}$, with $\lim _{t \rightarrow 0} \gamma(t)=0$, and $\gamma$ can be extended $C^{\infty}$ to $t=0$. Then, considering a modification $\gamma^{*}$ of $\gamma$ as we did in 2.2 and using Remarks 2.3 and 2.4, $\alpha$ is described in $\tilde{V}$ by $\gamma^{*}$ and it can be extended $C^{\infty}$ to $t=0$. Finally $\pi_{1} \circ \tilde{\pi} \circ \gamma^{*}$ is a curve which defines the ordering $\alpha$ and can be extended $C^{\infty}$ to $t=0$.

The second part comes from the first one, the above remark 3.0, and the fact that the set of orderings with maximum rank are dense (see [B], 8.4.9).

\section{REFERENCES}

[A] S. Abhyankar, Valuations centered in a local domain, Amer. J. Math., 78 (1956), 321-348.

[A-G-R] M. E. Alonso, J. M. Gamboa and J. M. Ruiz, Ordres sur les surfaces réelles, C. R. Acad. Sc. Paris t. 298, Série I, nº1 (1984), 17-19.

[An] C. Andradas, Real valuations in function fields, to appear in Comm. Algebra.

[B] G. W. Brumfiel, Partially Ordered Rings and Semi-Algebraic Geometry, London Math. Soc. Lect. Note Series 37. Cambridge Univ. Press (1979).

$[\mathrm{H}]$ D. Harrison, Finite and infinite primes for rings and fields, Mem. Amer. Math. Soc. $n^{\circ} 68,(1968)$.

[Hi] H. Hironaka, Resolution of singularities of an algebraic variety over a field of characteristic zero I, II, 79 (1964), 109-326.

[Pr] A. Prestel, Lectures on formally real fields, IMPA. Lecture Notes 22. Río de Janeiro (1975).

[R] J. M. Ruiz, Central orderings in fields of real meromorphic function germs, Manuscripta Math., 45 (1984), 193-214.

Received January 16, 1985 and in revised form July 1, 1985.

Departamento de Algebra y Fundamentos

UNIVERSIDAD COMPLUTENSE

28040 MADRID, SPAIN 



\section{PACIFIC JOURNAL OF MATHEMATICS EDITORS}

V. S. VARADARAJAN

(Managing Editor)

University of California

Los Angeles, CA 90024

HERBERT CLEMENS

University of Utah

Salt Lake City, UT 84112

R. FINN

Stanford University

Stanford, CA 94305
HERMANN FLASCHKA

University of Arizona

Tucson, AZ 85721

RAMESH A. GANGOLLI

University of Washington

Seattle, WA 98195

VAUGHAN F. R. JONES

University of California

Berkeley, CA 94720

ROBION KIRBY

University of California

Berkeley, CA 94720
C. C. MOORE

University of California

Berkeley, CA 94720

H. SAMELSON

Stanford University

Stanford, CA 94305

HAROLD STARK

University of California, San Diego

La Jolla, CA 92093

\section{ASSOCIATE EDITORS}

R. ARENS

E. F. BECKENBACH

B. H. NEUMANN (1906-1982)
F. WOLF

K. YOSHIDA

\section{SUPPORTING INSTITUTIONS}

UNIVERSITY OF ARIZONA

UNIVERSITY OF BRITISH COLUMBIA

CALIFORNIA INSTITUTE OF TECHNOLOGY

UNIVERSITY OF CALIFORNIA

MONTANA STATE UNIVERSITY

UNIVERSITY OF NEVADA, RENO

NEW MEXICO STATE UNIVERSITY

OREGON STATE UNIVERSITY
UNIVERSITY OF OREGON UNIVERSITY OF SOUTHERN CALIFORNIA

STANFORD UNIVERSITY

UNIVERSITY OF HAWAII

UNIVERSITY OF TOKYO

UNIVERSITY OF UTAH

WASHINGTON STATE UNIVERSITY

UNIVERSITY OF WASHINGTON 


\section{Pacific Journal of Mathematics}

\section{Vol. 123, No. $1 \quad$ March, 1986}

Maria Emilia Alonso García, A note on orderings on algebraic varieties $\ldots \ldots 1$

F. S. De Blasi and Józef Myjak, On continuous approximations for

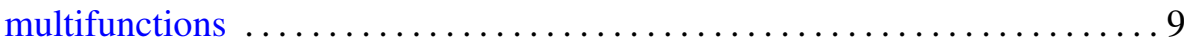

Frank Albert Farris, An intrinsic construction of Fefferman's CR metric . . . 33 Antonio Giambruno, P. Misso and Francisco César Polcino Milies, Derivations with invertible values in rings with involution $\ldots . \ldots . \ldots .47$

Dan Haran and Moshe Jarden, The absolute Galois group of a pseudo real

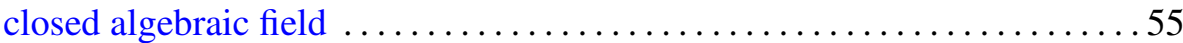

Telemachos E. Hatziafratis, Integral representation formulas on analytic

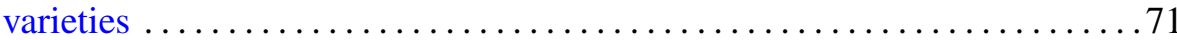

Douglas Austin Hensley, Dirichlet's theorem for the ring of polynomials

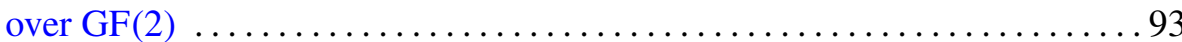

Sofia Kalpazidou, On a problem of Gauss-Kuzmin type for continued

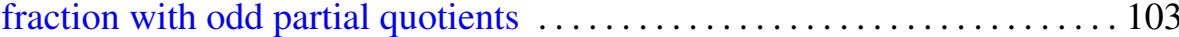

Harvey Bayard Keynes and Mahesh Nerurkar, Ergodicity in affine

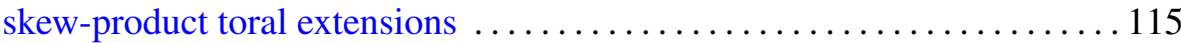

Thomas Landes, Normal structure and the sum-property $\ldots \ldots \ldots \ldots \ldots 127$

Anthony To-Ming Lau and Viktor Losert, Weak*-closed complemented invariant subspaces of $L_{\infty}(G)$ and amenable locally compact groups ...149 Andrew Lelek, Continua of constant distances in span theory . . . . . . . . 161 Dominikus Noll, Sums and products of $B_{r}$ spaces $\ldots \ldots \ldots \ldots \ldots \ldots \ldots \ldots$ Lucimar Nova, Fixed point theorems for some discontinuous operators 189

A. A. S. Perera and Donald Rayl Wilken, On extreme points and support points of the family of starlike functions of order $\alpha$

Massimo A. Picardello, Positive definite functions and $L^{p}$ convolution operators on amalgams ........................

Friedrich Roesler, Squarefree integers in nonlinear sequences ......... 223

Theodore Shifrin, The osculatory behavior of surfaces in $\mathbf{P}^{5}$ 